\title{
La gestión del agua depurada y la conservación del territorio y la biodiversidad. El caso de las lagunas de Campotejar y las Moreras en la Región de Murcia (SE España)
}

\section{Management of treated water and the conservation of territory and biodiversity. Case study Campotejar and Moreras in the region of Murcia, SE Spain}

\author{
Gustavo Alfonso Ballesteros Pelegrín ${ }^{1}$, Francisco Belmonte Serrato ${ }^{2}$ \\ y Daniel Ibarra Marinas ${ }^{3}$
}

\begin{abstract}
RESUMEN
Algunas depuradoras de lagunaje abandonadas, se han convertido en embalses de agua para uso agrícola, facilitando, en algunos casos, su transformación en humedales en los que se ha detectado la presencia de aves acuáticas amenazadas a escala mundial. Mediante el Proyecto LIFE09/NAT/000516 de la Unión Europea (2010-2014) se actuó en la adecuación paisajística y mejora ambiental en dos de estas lagunas (Lagunas de Campotejar y Lagunas de las Moreras) con el objetivo de transformarlas en humedales de Importancia Internacional (Sitios Ramsar). Los resultados demuestran que estas «lagunas» pueden cumplir por un lado, una función social y económica al actuar como reservorios de agua para regadío y potenciar el desarrollo de actividades de turismo de la naturaleza y educación ambiental; y, por otro, una función ecológica, como humedales de agua dulce o salobre donde se desarrollan hábitats de gran interés ecológico.
\end{abstract}

1 Departamento de Geografía, Universidad de Murcia gabpl@um.es ORCID iD: https:// orcid.org/0000-0001-8428-8379.

2 Departamento de Geografía, Universidad de Murcia franbel@um.es ORCID iD: https:// orcid.org/0000-0002-1331-1743.

3 Departamento de Geografía, Universidad de Murcia adaniel.ibarra@um.es https://orcid. org/0000-0003-3683-4456.

Copyright: (C) 2018 CSIC. Este es un artículo de acceso abierto distribuido bajo los términos de la licencia de uso y distribución Creative Commons Reconocimiento 4.0 Internacional (CC BY 4.0). 
Palabras Clave: depuradoras de lagunaje; Ramsar; uso social; función ecológica; conservación.

\begin{abstract}
Some abandoned sewage lagoons have become agricultural reservoirs, easing in some cases transformation into wetlands in which the presence of endangered aquatic birds has been detected. By way of the European Union Project LIFE09/NAT/000516 landscape adaption and environmental improvement in two of these lagoons (Lagunas de Campotejar y Lagunas de las Moreras) with the objective of transforming them into wetlands of international importance (Ramsar Convention). The results demonstrate these "lagoons" can fulfil on the one hand a social and economic role by acting as reservoirs for irrigation strengthen nature tourism and environmental education. And on the other hand an ecological role as freshwater or brackish wetlands where habitats of great ecological interest can develop.
\end{abstract}

KEY WORDS: sewage lagoons; Ramsar; social use; ecological role; conservation.

Como citar este artículo / Citation: Ballesteros Pelegrín, G. A., Belmonte Serrato, F. e Ibarra Marinas, D. (2018): "La gestión del agua depurada y la conservación del territorio y la biodiversidad. El caso de las lagunas de Campotejar y las Moreras en la Región de Murcia (SE España)", Estudios Geográficos, LXXIX/285, pp. 397-417. https://doi. org/10.3989/estgeogr.201815.

\title{
INTRODUCCIÓN
}

El agua es la base de la vida. Es un recurso crucial para la humanidad, que genera y sostiene la prosperidad económica y social. También es un elemento central de la regulación natural de los ecosistemas y el clima.

La calidad del agua se ve amenazada por la contaminación, sobreexplotación y los cambios hidromorfológicos impuestos por la industria, la agricultura, el desarrollo urbano, las defensas contra las inundaciones, la generación de electricidad, la navegación, los usos recreativos o la descarga de aguas residuales.

La política ambiental europea tiene entre sus principios fundamentales, la conservación, protección y mejora de la calidad del agua así como la utilización prudente y racional de los recursos naturales (Artículo 130R del Tratado de la Unión Europea).

Para la consecución de dichos objetivos se promovió la Directiva Marco del Agua (Directiva 2000/60/EC), que ha marcado las líneas en las que se han apoyado diferentes estrategias a lo largo del tiempo: desde la protección de 
los recursos hídricos en función de los usos del agua, al control de vertidos mediante normas de emisión para llegar a una estrategia ambiental basada en la protección de las masas de agua, consideradas estas como ecosistemas acuáticos, dando un enfoque más ambiental que promueve e impulsa un uso sostenible del agua. Por otro lado, para la protección de la biodiversidad y el territorio que la sustenta, se aprobaron la Directiva 2009/147/CE de 30 de noviembre de 2009 relativa a la conservación de las aves silvestres ${ }^{4}$ y la Directiva 1992/43/CEE relativa a la conservación de los hábitats naturales y de la fauna y flora silvestres 5 .

El Primer Plan Nacional de Saneamiento y Depuración 1995-2005, desarrollado en España, produjo un cambio relevante, derivado de la aprobación y transposición de la Directiva CEE 91/271, sobre saneamiento y depuración, que aporta un nuevo enfoque integrado de la política de aguas. Posteriormente, el Gobierno de España redactó un segundo Plan Nacional de Calidad de las Aguas: Saneamiento y Depuración 2007-2015, que daba respuesta, tanto a los objetivos no alcanzados por el anterior Plan, como a las nuevas necesidades planteadas por la Directiva Marco del Agua. Este nuevo Plan Nacional de Calidad de las Aguas formó parte de un conjunto de medidas para alcanzar el buen estado ecológico de las masas de agua, que la Directiva Marco del Agua propugna para conseguir un desarrollo socioeconómico equilibrado y ambientalmente sostenible. Uno de los retos del Plan, fue generalizar sistemas eficaces de depuración y redes de saneamiento que llevaran todas las aguas residuales a depuradoras en todas las aglomeraciones urbanas de más de 2.000 habitantes y que sustituyeran los antiguos sistemas de lagunaje por otros ambientalmente menos perjudiciales.

Según las estimaciones del Plan Nacional de Calidad de las Aguas vigente, en la actualidad existen en España más de 2.500 Estaciones Depuradoras de Aguas Residuales (EDAR), que depuran más de $3.375 \mathrm{hm}^{3}$ anuales de aguas residuales. De éstas, se estima que se reutilizan unos $450 \mathrm{hm}^{3} / \mathrm{año}$, lo que supone poco más del 13\% del total. La distribución por usos del agua depurada se sitúa en unas tres cuartas partes para regadío agrícola, alrededor del 12\% para usos recreativos y campos de golf, el 6\% para servicios urbanos, el $4 \%$ para usos ecológicos, caudal ecológico de ríos y recarga de de acuíferos, y un 3\% para uso industrial (Ministerio de Medio Ambiente, 2007).

En la Región de Murcia se construyeron en la primera década del siglo XXI modernas estaciones depuradoras de aguas residuales urbanas con

4 Disponible en: http://eur-lex.europa.eu/legal-content (Fecha de consulta: 26/3/2017).

5 Disponible en: http://eur-lex.europa.eu/legal-content (Fecha de consulta: 26/3/2017) 
alta tecnología de depuración, que sustituyeron a obsoletas estaciones que utilizaban el sistema de lagunaje, construidas a lo largo de los años 80 del siglo XX. Según la Entidad de Saneamiento y Depuración de Aguas Residuales de la Región de Murcia (Esamur, 2015), en diciembre de 2014 en la Región de Murcia se depuran el agua de 118 aglomeraciones urbanas con un total de 1.343.963 habitantes, que generan un volumen de $115,08 \mathrm{hm}^{3}$ de aguas residuales, que representa la purificación del $99,1 \%$ de la población.

Algunas de las nuevas depuradoras fueron construidas junto a las antiguas balsas de lagunaje, que son utilizadas como depósitos, para uso principalmente agrícola, de un agua que previamente ha sido depurada.

Algunos de estos espacios se han convertido en humedales que sirven de refugio de vida silvestre, sobre todo aves acuáticas, gracias a la mejora calidad de sus aguas y el desarrollo de vegetación perilagunar, con la presencia de especies amenazadas a escala mundial como son la malvasía cabeciblanca (Oxyura leucocephala), la cerceta pardilla (Marmaronetta angustirostris) y el porrón pardo (Aythya niroca). Cabe destacar que la población mundial de malvasía cabeciblanca es inferior a 10.000 ejemplares, de los que unos 3.000 ejemplares se encuentran en el mediterráneo occidental: España, Marruecos, Argelia y Túnez (Madroño, González y Atienza, 2005 y Crivelli, 1994), por tanto, estos enclaves pueden ser considerados como modelo de desarrollo sostenible, donde se compatibiliza la depuración de agua residual urbana, la conservación del territorio y la biodiversidad, con el posterior uso del agua, principalmente para la agricultura (Ballesteros y Muñoz, 201la y 2011b).

De ésta manera, la depuración de agua residual en modernas depuradoras y su almacenamiento en los antiguos estanques de lagunaje, previo a su uso para riego agrícola, pone en valor una infraestructura verde, generando nuevos servicios ecosistémicos, donde permite cerrar el ciclo de depuración de agua, conservación una porción del territorio y uso del agua para regadío.

La interacción entre depuración de agua-conservación del territorio y la biodiversidad-uso del agua para irrigación, fue valorada de forma positiva por la Unión Europea, que en 2010 aceptó financiar en un 75\% el Proyecto LIFE09/NAT/000516 presupuestado con 1.342.103 , para desarrollar, entre otras actuaciones la adecuación paisajística y mejora ambiental en dos antiguas depuradoras de lagunaje: Lagunas de las Moreras (Mazarrón) y Lagunas de Campotejar (Molina de Segura). Los valores naturales de las lagunas de las Moreras y Campotejar motivaron su declaración como Humedales de Importancia Internacional del Convenio Ramsar (Boletín Oficial del Estado n. ${ }^{\circ}$ 30 de 4 de febrero de 2011). 


\section{OBJETIVO y METODOLOGÍA}

El objetivo de este trabajo es poner de manifiesto que las antiguas depuradoras de lagunaje pueden ser adecuadas como reservorios de agua y humedales, capaces de ejercer una importante función social y ecológica, modelo de creación de nueva infraestructura verde sostenible y compatible con la conservación de la naturaleza.

La metodología empleada para adecuar las lagunas artificiales que reciben el agua depurada y que el conjunto cumpla la tripe función (depuración-conservación-riego), se basó en un conocimiento previo del territorio y sus valores naturales; generar una actitud positiva de los diferentes actores e implicarlos de forma activa: administración local (ayuntamientos de Molina de Segura y Mazarrón), Comunidad Autónoma de la Región de Murcia, Confederación Hidrográfica del Segura, empresa responsable de la depuración del agua y comunidades de regantes; obtención de fondos europeos para el desarrollo del Proyecto y generar una normativa de conservación que regule los usos.

\section{Resultados}

Función social, económica y ambiental de las lagunas

Lagunas de las Moreras

El Sitio Ramsar de las lagunas de las Moreras tienen una superficie de 72,3 ha localizadas en el sureste de la Península Ibérica, al sur de la Comunidad Autónoma de la Región de Murcia, en el Término Municipal de Mazarrón. Está formado por la rambla de las Moreras desde del puente de acceso a Mazarrón hasta la desembocadura en el Mar Mediterráneo. Dispone de 2 lagunas, una situada en la antigua depuradora de aguas residuales de lagunaje y la otra junto al cauce de la rambla de las Moreras (figura 1) que almacenan agua de forma permanente por los aportes de una moderna planta de aguas residuales que la vierte previamente depurada.

Se encuentra en un área climática árida-semiárida, con precipitación media anual de unos 200-300 mm, con el máximo pluviométrico a finales de otoño y un largo periodo de sequía estival. La temperatura media anual es de $17-19^{\circ} \mathrm{C}$, con una evapotranspiración potencial media de $900 \mathrm{~mm}$ y un alto déficit hídrico anual por el alto índice de insolación de más de 2.500 horas anuales. 


\section{FIGURA 1}

LOCALIZACIÓN DEL SITIO RAMSAR DE LAS LAGUNAS DE LAS MORERAS
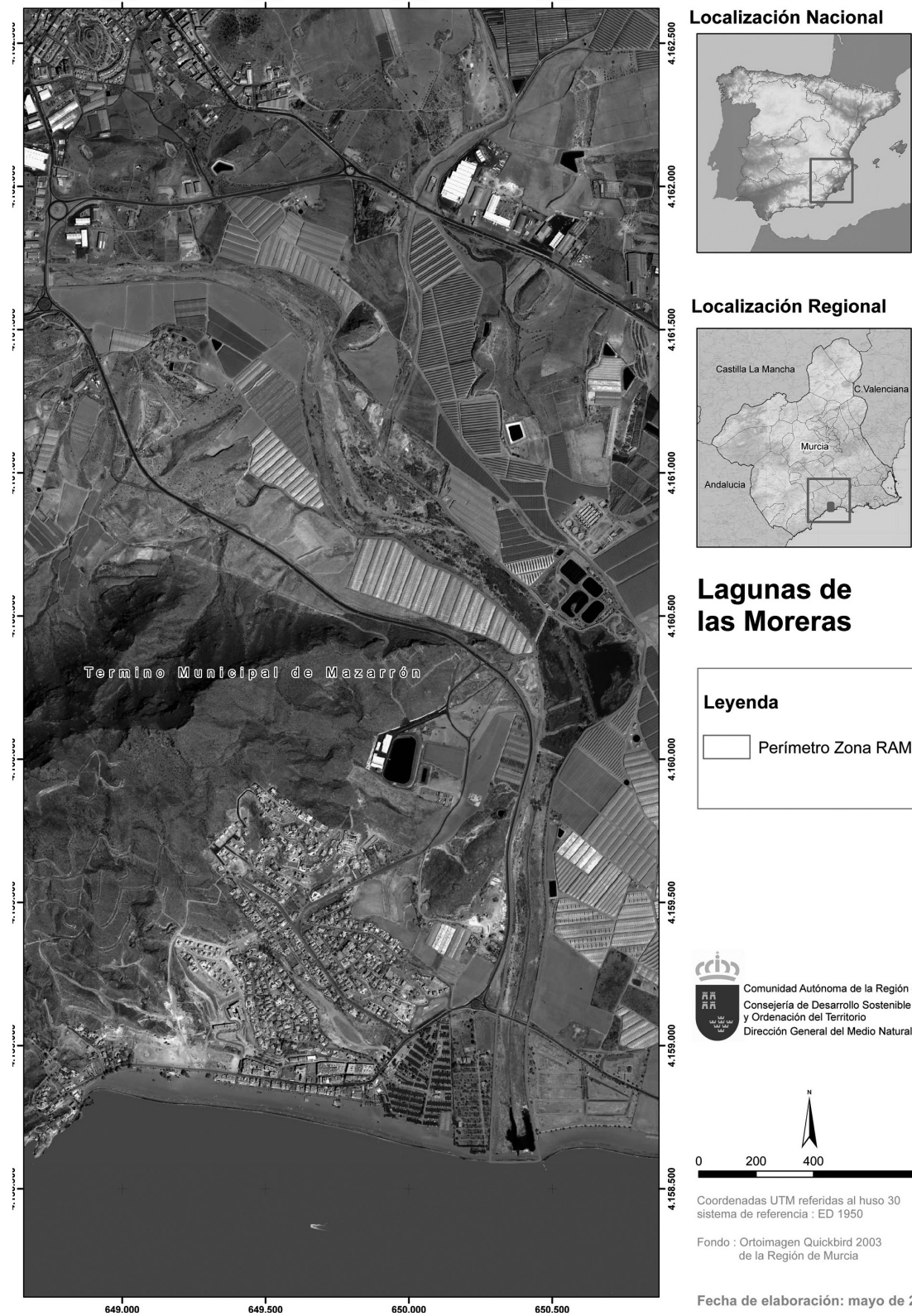

Localización Regional

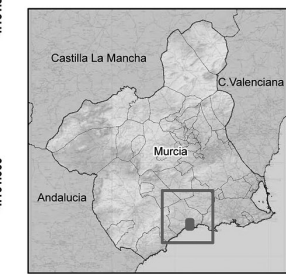

\section{Lagunas de las Moreras}

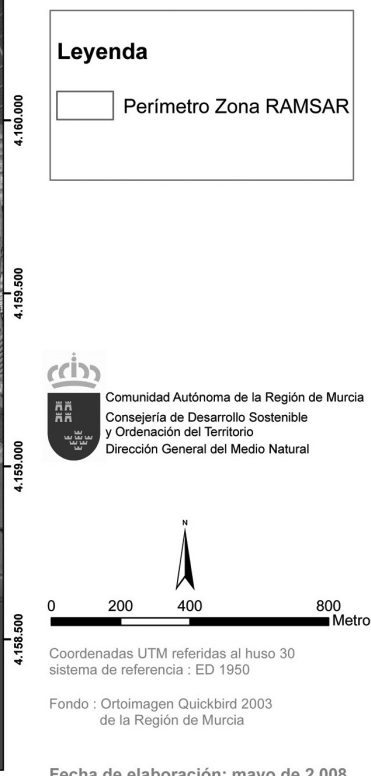

Fuente: Ballesteros y Muñoz (2011b).

Estudios Geográficos, Vol. LXXIX, 285, pp. 397-417, julio-diciembre 2018

ISSN: 0014-1496, eISSN: 1988-8546, doi: https//doi.org/10.3989/estgeogr.201815 
La rambla de las Moreras discurre sobre materiales metamórficos del PermoTriásico y margosos y volcánicos del Neógeno. Estos proceden de depósitos aluviales aportados por la rambla, enmarcados por formaciones de derrubios de ladera que se asientan en la base de la Sierra de las Moreras. El cauce de la rambla de las Moreras discurre encajonado entre taludes con escasa vegetación en su cabecera y con mayor densidad en los últimos $2 \mathrm{~km}$. El sustrato se caracteriza por el predominio de margas miocénicas y del Trías y la presencia de sedimentos finos (limos y arcillas). En sus orillas se ha desarrollado una extensa e inaccesible orla de carrizal y tarayal. En la cabecera de las lagunas se desarrollan formaciones halófilas típicas del mediterráneo español, que incluyen diversos hábitats de interés comunitario.

Las lagunas se han convertido en un lugar importante de invernada, migración y reproducción de numerosas especies de aves acuáticas entre las que destaca, por su grado de amenaza mundial, la nidificación de la Malvasía cabeciblanca (Oxyura leucocephala) (Ballesteros, 2015) y la Cerceta pardilla (Marmaronetta angustirostris) y por amenazar en el contexto europeo el Porrón pardo (Aythya nyroca), presente durante el invierno. El Sitio Ramsar está formado además por el cauce de la rambla de las Moreras desde el puente de las Moreras hasta la desembocadura.

La EDAR mediante el sistema de lagunaje de Mazarrón estuvo funcionando en la década de los 90, cesando su actividad en 2005, cuando se pone en marcha la nueva EDAR, con una capacidad de depuración de $15.000 \mathrm{~m}^{3} /$ día, que puede dar servicio a una población de unos 15.000 habitantes (Esamur, 2015).

El agua residual se depura aplicando una tecnología de Fangos Activos - Aireación Prolongada + Coagulación + Floculación + Filtro de Anillas + Desinfección Ultravioleta, lo que permite obtener un rendimiento de depuración del 98,60\% de sólidos en suspensión, del 94,50\% de DQO y del $98,80 \%$ de $\mathrm{DBO}_{5}$ (Esamur, 2015)

Actualmente hay en funcionamiento dos líneas de depuración independientes (Figura 2) cuyos efluentes vierten a balsas almacenadoras diferentes (Esamur, 2015):

1. Agua residual del pueblo de Mazarrón, con escaso grado de salinidad que, tras su depuración es derivada directamente a la balsa de la Comunidad de Regantes de Mazarrón.

2. Agua residual del Puerto de Mazarrón, que por su elevado grado de salinidad no es apta para uso agrario, por lo que tras su depuración, circula por la balsa de la antigua depuradora de lagunaje y después es vertida al humedal de la Gravera de las Moreras, cuyo aliviadero vierte a la rambla de las Moreras. 
La ubicación de este espacio protegido en zonas de alto interés turístico, ha favorecido un aumento del turismo de deporte y naturaleza, donde destaca el interés por la observación y fotografía de aves.

\section{FIGURA 2}

\section{CIRCULACIÓN DEL AGUA DULCE Y DEL AGUA SALOBRE}

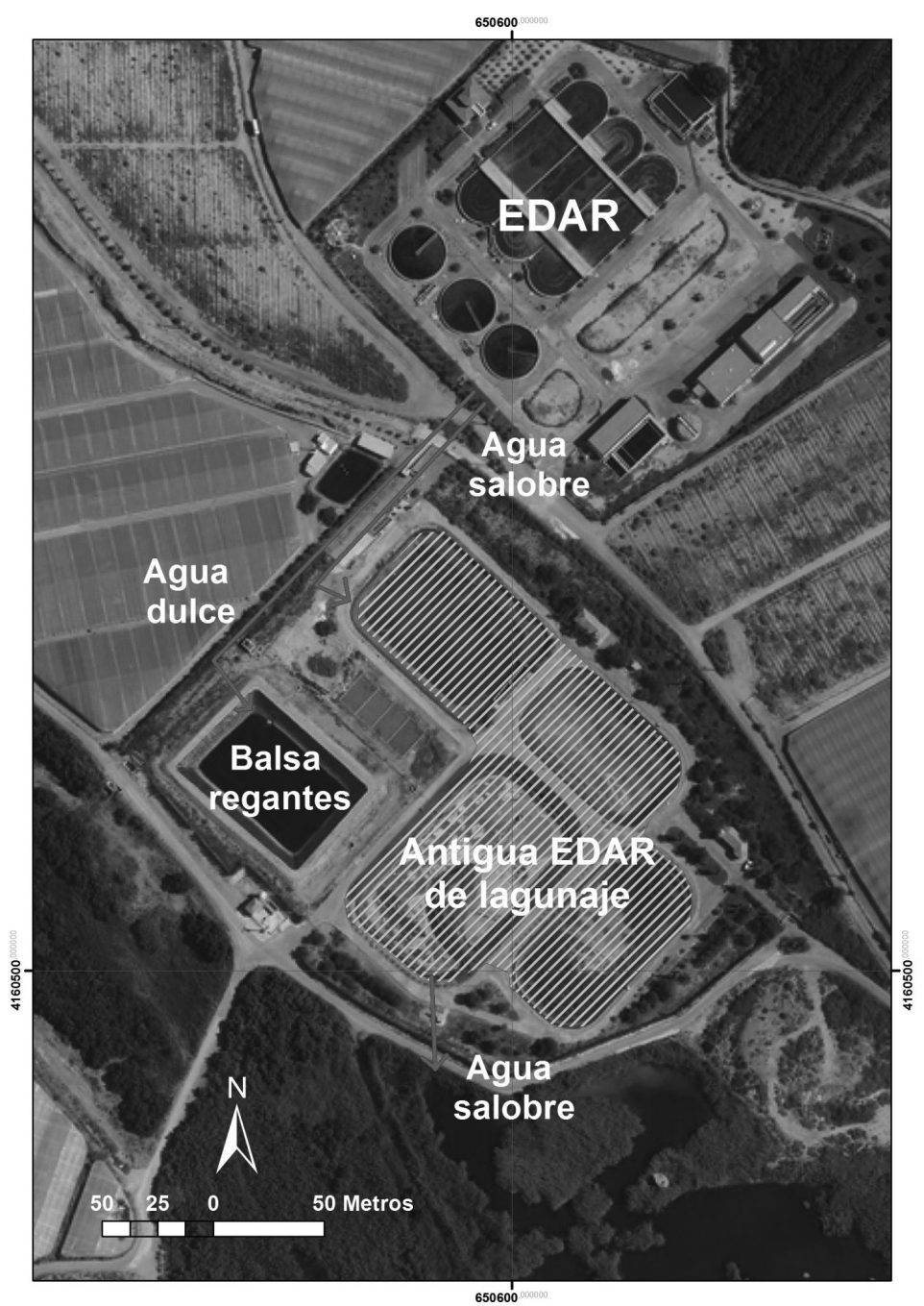

Fuente: Elaboración propia.

Estudios Geográficos, Vol. LXXIX, 285, pp. 397-417, julio-diciembre 2018

ISSN: 0014-1496, eISSN: 1988-8546, doi: https//doi.org/10.3989/estgeogr.201815 
Las lagunas de las Moreras cumplen 3 de los 9 criterios que establece la Convención de Ramsar para declaración de Humedales de Importancia Internacional:

Criterio 2 (si sustenta especies vulnerables, en peligro o en peligro crítico, o comunidades ecológicas amenazadas). En el caso de España, un humedal cumple este Criterio cuando presenta especies y/o hábitat asociados a ambientes húmedos amenazados en un contexto biogeográfico supranacional, por ejemplo taxones clasificados en las máximas categorías de amenaza de UICN, Catálogo ó Libros Rojos Nacionales, etc., y/o hábitat prioritarios del Anexo I de la Directiva de Hábitat, etc. (Comité de Humedales, 2011).

En las lagunas de Campotejar se han citado 15 especies asociadas a ambientes húmedos con un alto grado de amenaza entre las que destacan las siguientes especies muy amenazadas y/o incluidas en el anexo I de la Directiva Aves o en el anexo II de la Directiva Hábitats (Tabla 1).

Criterio 3 (si sustenta poblaciones de especies vegetales y/o animales importantes para mantener la diversidad biológica de una región biogeográfica determinada). En el caso de España, un humedal cumple el criterio 3 cuando presenta un número apreciable de endemismos y/o una gran riqueza específica de taxones asociados a ambientes húmedos (Comité de Humedales, 2011).

TABLA 1

ESPECIES DE VERTEBRADOS AMENAZADADOS EN LAS LAGUNAS DE LA MORERAS

\begin{tabular}{|c|c|c|c|c|}
\hline Taxón & $\begin{array}{l}\text { UICN } \\
(2011)\end{array}$ & $\begin{array}{c}\text { Directiva } \\
\text { Hábitat } \\
\text { (92/43/ } \\
\text { CEE) } \\
\end{array}$ & $\begin{array}{c}\text { Catálogo Español } \\
\text { de Especies } \\
\text { Amenazadas } \\
\text { (RD 139/2011) }\end{array}$ & $\begin{array}{c}\text { Libros Rojos } \\
\text { nacionales }\end{array}$ \\
\hline Fartet Aphanius iberus & Vulnerable & Anexo II & En Peligro & En Peligro \\
\hline Galápago leproso Mauremys leprosa & & Anexo II-IV & & Vulnerable \\
\hline Garcilla cangrejera Ardeola ralloides & & & En Peligro & \\
\hline Cerceta pardilla Marmaronett.angustirostris & Vulnerable & & En Peligro & En Peligro Crítico \\
\hline Porrón pardo Aythya nyroca & & & En Peligro & En Peligro Crítico \\
\hline Malvasía cabeciblanca Oxyura leucocephala & En Peligro & & En Peligro & En Peligro \\
\hline Archibebe común Tringa totanus & & & & Vulnerable \\
\hline Fumarel común Chlidonias níger & & & En Peligro & Vulnerable \\
\hline Fumarel cariblanco Chlidonias hybridus & & & & Vulnerable \\
\hline Carricerín real Acrocephalus melanopogon & & & & Vulnerable \\
\hline
\end{tabular}

Fuente: Elaboración propia a partir de Ballesteros y Muñoz (2011b). 
En las lagunas de las Moreras se citan 3 especies endémicas de invertebrados, de las cuales una es exclusiva de la Península Ibérica: Nebrioporus baeticus y 2 son endemismos iberoafricanos: Ochthebius cuprescens y Ochthebius tacapasensis (Moreno et al., 1997 y Sánchez-Fernández et al., 2003).

Criterio 6 (si sustenta de manera regular el $1 \%$ de los individuos de una población, especie o subespecie de ave acuática). En el caso de España se considera que un humedal cumple este criterio cuando sustenta de una manera regular el 1\% (según Wetlands International, 2006) de los individuos de una población biogeográfica de una especie de ave acuática determinada (Comité de Humedales, 2011). Las lagunas de las Moreras sustentan desde 2005 manera regular más del 1\% de la población del Mediterráneo occidental de malvasía cabeciblanca (O. leucocephala) (Tabla 2).

TABLA 2

NÚMERO ANUAL MÁXIMO DE MALVASÍA CABECIBLANCA EN LAS LAGUNAS DE LAS MORERAS

\begin{tabular}{c|c|c|c|c|c|c|c|c|c|c|c|c|c}
\hline $\begin{array}{c}\text { Criterio 1\% } \\
\begin{array}{c}\text { Wetlands } \\
\text { Internacional } \\
(2006)\end{array}\end{array}$ & 2004 & 2005 & 2006 & 2007 & 2008 & 2009 & 2010 & 2011 & 2012 & 2013 & 2014 & 2015 & 2016 \\
\hline 25 ejemplares & 1 & 29 & 61 & 43 & 39 & 35 & 27 & 22 & 21 & 18 & 40 & 66 & 26 \\
\hline
\end{tabular}

Fuente: Elaboración propia a partir de Ballesteros y Muñoz (2011b) y Ballesteros (2015).

\section{Lagunas de Campotejar}

Las Lagunas de Campotejar se localizan en el sureste de la Península Ibérica (Región de Murcia), en los Términos Municipales de Molina de Segura y Lorquí, y abarca una extensión de 61 ha (Esamur, 2015).

Integra en sus límites la cabecera de la rambla del Salar Gordo y cinco balsas artificiales (Figura 3), construidas inicialmente para la depuración del agua residual de Molina de Segura, pero actualmente almacenan agua para uso agrícola, agua que es previamente tratada (fangos activos, de doble etapa y tratamiento terciario) con una capacidad de depuración diaria de $23.280 \mathrm{~m}^{3}$. El complejo lagunar se encuentra muy naturalizado por el desarrollo de un cinturón de carrizo en gran parte de sus orillas.

Se asientan sobre una depresión margosa de formas aplanadas, ocupada anteriormente por estepas salinas en su mayor parte desaparecidas. El relieve se caracteriza por la presencia de formas muy aplanadas propias del final de un 
glacis, que contrastan con las pequeñas lomas terciarias y margosas adyacentes. La morfología se encuentra muy alterada por la actividad antrópica.

La característica esencial de estos suelos es su alta salinidad y en la que la dinámica de las sales solubles ha conducido a una mayor diferenciación del perfil. En superficie presentan eflorescencias salinas y en profundidad numerosas manchas ocres y rojizas. Están ocupados por vegetación halófila de gran interés ecológico y son improductivos.

Las temperaturas máximas se registran en agosto, con $26,2^{\circ} \mathrm{C}$ de media, el resto del período permanecen algo más suaves, siendo la mínima en enero con $9,8^{\circ} \mathrm{C}$. Las precipitaciones, al igual que las temperaturas, presentan cierta irregularidad, siendo los meses de mayor precipitación los de octubre (media 49,7 $\mathrm{mm}$ ) y mayo (media $38,7 \mathrm{~mm}$ ). El periodo seco viene representado por los meses de julio y agosto, cuando la precipitación adquiere el valor de 4 y $9 \mathrm{~mm}$ de media, respectivamente.

FIGURA 3

LOCALIZACIÓN DEL SITIO RAMSAR DE LAS LAGUNAS DE CAMPOTEJAR

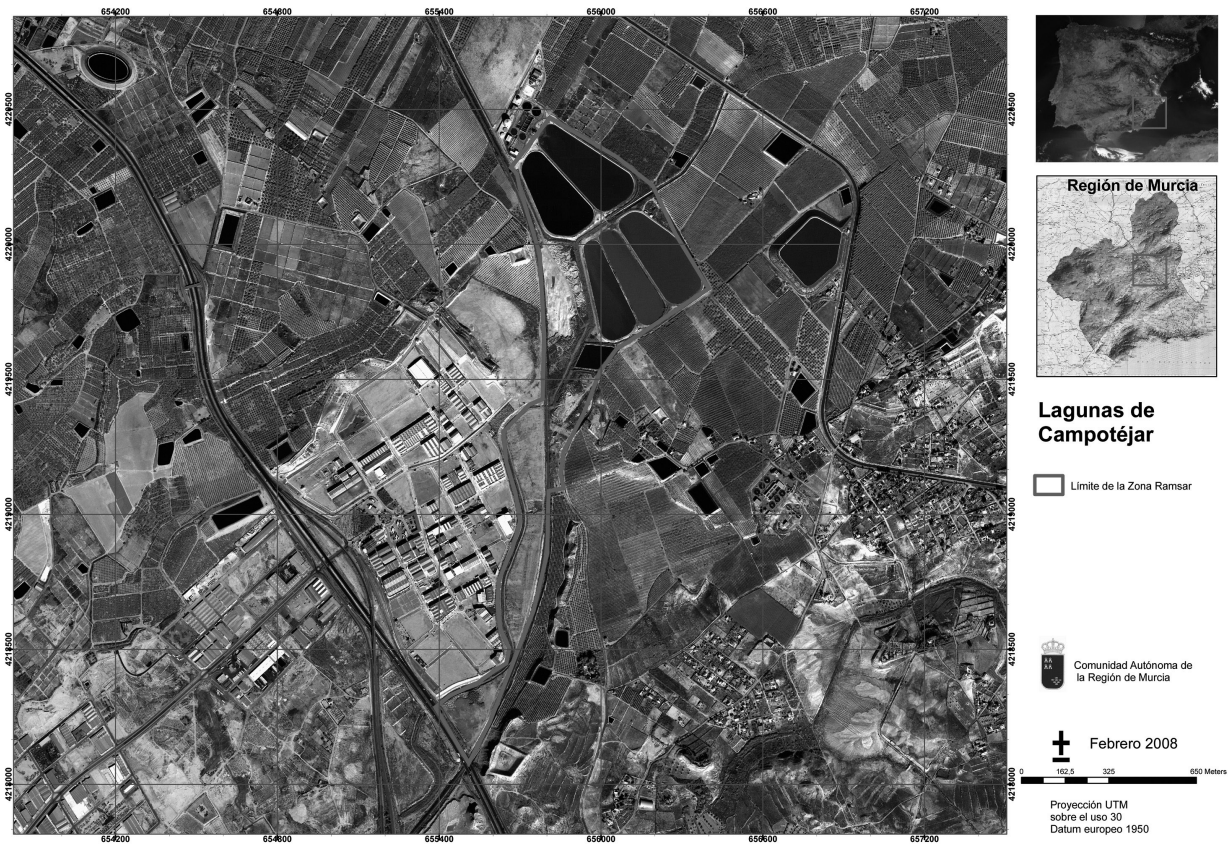

Fuente: Ballesteros y Muñoz (201la).

Estudios Geográficos, Vol. LXXIX, 285, pp. 397-417, julio-diciembre 2018 ISSN: 0014-1496, eISSN: 1988-8546, doi: https//doi.org/10.3989/estgeogr.201815 
Las lagunas de las Moreras cumplen 2 de los 9 criterios que establece la Convención de Ramsar para declaración de Humedales de Importancia Internacional (Ballesteros y Muñoz, 2011a):

Criterio 2 (si sustenta especies vulnerables, en peligro o en peligro crítico, o comunidades ecológicas amenazadas). Está citada la presencia de 13 especies de aves asociadas a ambientes húmedos con un alto grado de amenaza (Tabla 3), entre los que destacan las siguientes especies con mayor grado de amenaza (Ballesteros y Muñoz, 2011b).

Entre los tipos de hábitat asociados a ambientes húmedos del Anexo I de la Directiva Hábitat 1992/43/CE, destaca la existencia del hábitat prioritario $1510^{*}$ Estepas salinas mediterráneas (Limonietalia) (Ballesteros y Muñoz, 2011b).

TABLA 3

ESPECIES DE VERTEBRADOS AMENAZADOS

DE LAS LAGUNAS DE CAMPOTÉJAR

\begin{tabular}{l|c|c|c}
\hline \multicolumn{1}{c|}{ Taxón } & UICN (2011) & $\begin{array}{c}\text { Catálogo Español de } \\
\text { Especies Amenazadas } \\
\text { (RD 139/2011) }\end{array}$ & $\begin{array}{c}\text { Libros Rojos } \\
\text { nacionales }\end{array}$ \\
\hline Garcilla cangrejera Ardeola ralloides & & En Peligro & En Peligro \\
\hline Cerceta pardilla Marmaronett.angustirostris & Vulnerable & En Peligro & En Peligro Crítico \\
\hline Porrón pardo Aythya nyroca & & En Peligro & En Peligro \\
\hline Malvasía cabeciblanca Oxyura leucocephala & En Peligro & Vulnerable \\
\hline Pagaza piconegra Gelochelidon nilotica & & & Vulnerable \\
\hline Fumarel cariblanco Chlidonias hybridus & & & En Peligro \\
\hline Fumarel común Chlidonias niger & & & Vulnerable \\
\hline Carricerín real Acrocephalus melanopogon & & & \\
\hline
\end{tabular}

Fuente: Elaboración propia a partir de Ballesteros y Muñoz, (2011 a).

Criterio 6 (si sustenta de manera regular el 1\% de los individuos de una población, especie o subespecie de aves acuáticas). Las lagunas de Campotejar sustentan desde 2005 más del 1\% de la población del Mediterráneo occidental de malvasía cabecbiblanca (O. leucocephala) (tabla 4). 
TABLA 4

Na ANUAL MÁXIMO DE MALVASÍA CABLECIBLANCA EN LAGUNAS DE CAMPOTÉJAR

\begin{tabular}{c|c|c|c|c|c|c|c|c|c|c|c|c|c}
\hline $\begin{array}{c}\text { Criterio 1\% } \\
\text { Wetlands } \\
\begin{array}{c}\text { Internacional } \\
(2006)\end{array}\end{array}$ & 2004 & 2005 & 2006 & 2007 & 2008 & 2009 & 2010 & 2011 & 2012 & 2013 & 2014 & 2015 & 2016 \\
\hline 25 ejemplares & 15 & 243 & 224 & 78 & 195 & 157 & 209 & 154 & 130 & 136 & 60 & 51 & 70 \\
\hline
\end{tabular}

Fuente: Elaboración propia a partir de Ballesteros y Muñoz (2011a) y Ballesteros (2015).

\section{Actuaciones sobre el paisaje y el hábitat}

\section{Lagunas de las Moreras}

En el marco del Proyecto LIFE09/NAT/000516 se ejecutaron actuaciones en la antigua depuradora de lagunaje de Mazarrón, que estaba abandonada. El objetivo era acondicionar los 4 antiguos estanques de lagunaje de la depuradora (Figura 4) para crear una única laguna, consiguiendo el aumento de hábitat de vertebrados asociados a humedales y mejora la calidad paisajística. Antes del inicio de las excavaciones, se procedió a la retirada de los restos presentes en las antiguas balsas de lagunaje que correspondían a tuberías, aireadores, pasarela, redes, barandillas y otros residuos

\section{FIGURA 4}

DEPURADORA ABANDONADA DE MAZARRÓN. ESTADO INICIAL DE LAS OBRAS

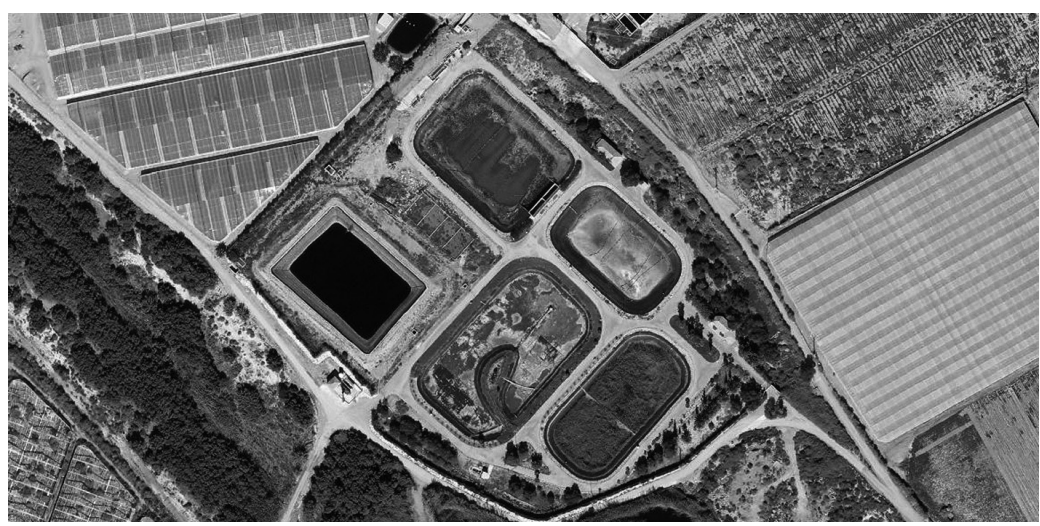

Fuente: Cartomur. Ortofoto de 2009 (http://cartomur.imida.es/visorcartoteca/).

Estudios Geográficos, Vol. LXXIX, 285, pp. 397-417, julio-diciembre 2018 ISSN: 0014-1496, eISSN: 1988-8546, doi: https//doi.org/10.3989/estgeogr.201815 
Posteriormente se llevaron a cabo las demoliciones y excavaciones de las motas que separaban los 4 estanques de la antigua depuradora de Mazarrón (Figura 5) para la unificación y acondicionamiento de una laguna con una superficie total de $19.000 \mathrm{~m}^{2}$ y un volumen de agua de unos $53.000 \mathrm{~m}^{3}$.

\section{FIGURA 5}

\section{VISTA DE LAS BALSAS QUE SE UNIFICARON}

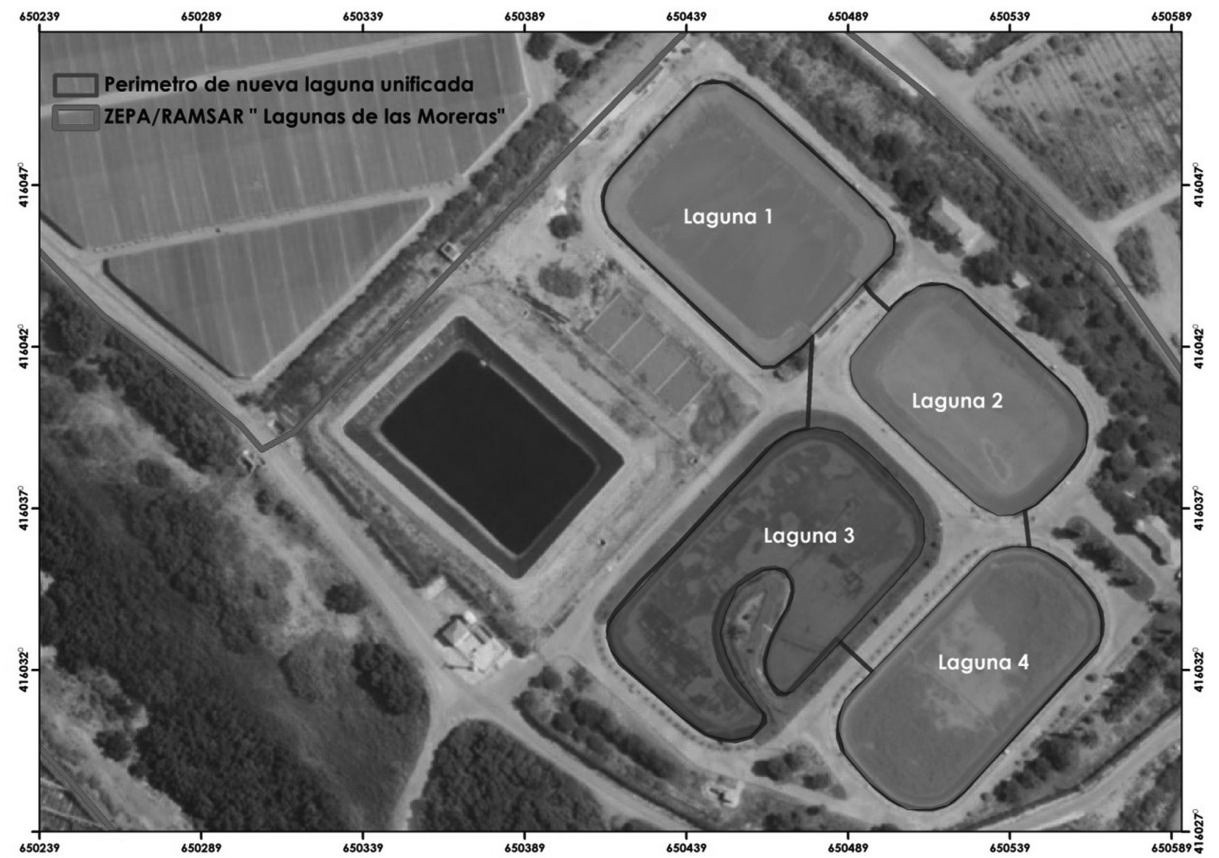

Fuente: fondo documental de la Dirección General de Medio Ambiente.

Tras eliminar los residuos, se iniciaron las demoliciones de las motas existentes entre las balsas de lagunaje con ayuda de un retro-martillo rompedor (Figura 6).

Posteriormente, se inició la excavación y movimientos de tierra pertinentes en las 4 motas existentes que habían sido demolidas anteriormente. Todos los movimientos de tierra se efectuaron teniendo en cuenta la cota del nivel de agua en cada una de las balsas, conforme a los datos del levantamiento topográfico previo realizado en la zona, perfilando una única charca o laguna diseñada por el proyecto de ejecución. 
La tierra excavada para la unificación de la laguna fue reutilizada principalmente para la creación de una mota de $328 \mathrm{~m}$ de recorrido sobre una superficie de $1.640 \mathrm{~m}^{2}$ y con una altura media de $1 \mathrm{~m}$ en la zona sur de la nueva laguna, y a su vez, para el perfilado de la laguna, creando montículos y formas irregulares para conseguir dotar al terreno de un cierto grado de naturalidad.

\section{FIGURA 6}

\section{IMAGEN DE LA ESTRUCTURA DE HORMIGON DEMOLIDA}

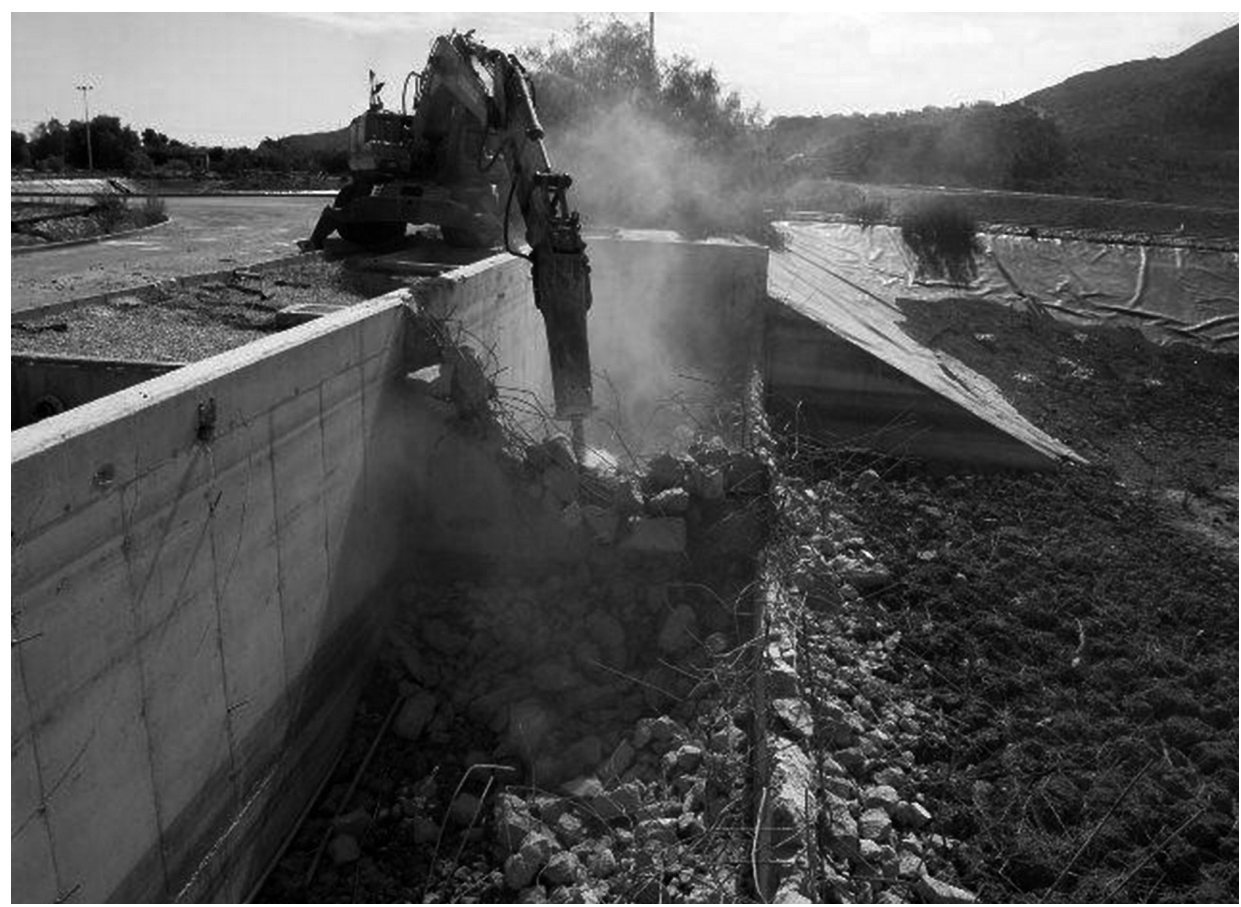

Fuente: fondo documental de la Dirección General de Medio Ambiente. Eliminación del hormigón de las motas de separación de los estanques para la creación de una sola laguna.

Una vez finalizado la unificación de las balsas y el acondicionamiento de la laguna mediante los movimientos de tierra pertinentes, se procedió a la impermeabilización (Figura 7) de las zonas desprovistas de lámina geo-textil, (superficie donde se realizaron los principales movimientos de tierras y donde la lámina que estaba presente tuvo que ser retirada) y en aquellos puntos concretos donde la lámina estaba deteriorada. 
Para el abastecimiento de agua desde la EDAR de Mazarrón-Norte hasta la nueva laguna, se llevó a cabo la instalación de una tubería por la que circula el agua salobre procedente del Puerto de Mazarrón.

\section{FIGURA 7}

\section{ESTADO DE LA LAGUNA DESPUÉS DE LA UNIFICACIÓN DE LOS 4 ESTANQUES LAGUNARES}

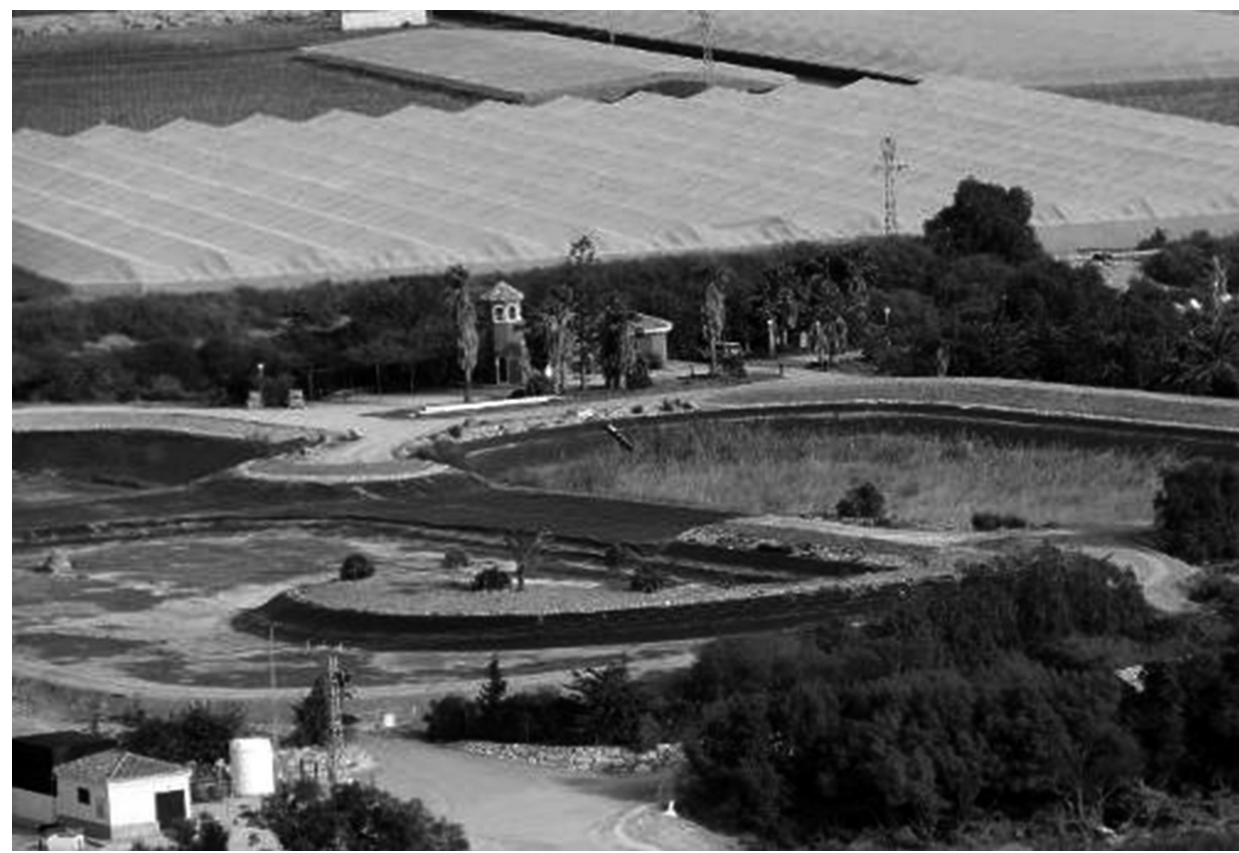

Fuente: archivo Dirección General de Medio Ambiente de la Región de Murcia.

Como resultado de la unificación de las 4 balsas de lagunaje de la antigua depuradora se consiguió aumentar la superficie del humedal de Lagunas de las Moreras alrededor de $19.000 \mathrm{~m}^{2} \mathrm{y}$ un volumen de agua de unos $53.000 \mathrm{~m}^{3}$ (Figura 8). 


\section{Figura 8}

\section{ESTADO FINAL DE LA LAGUNA}

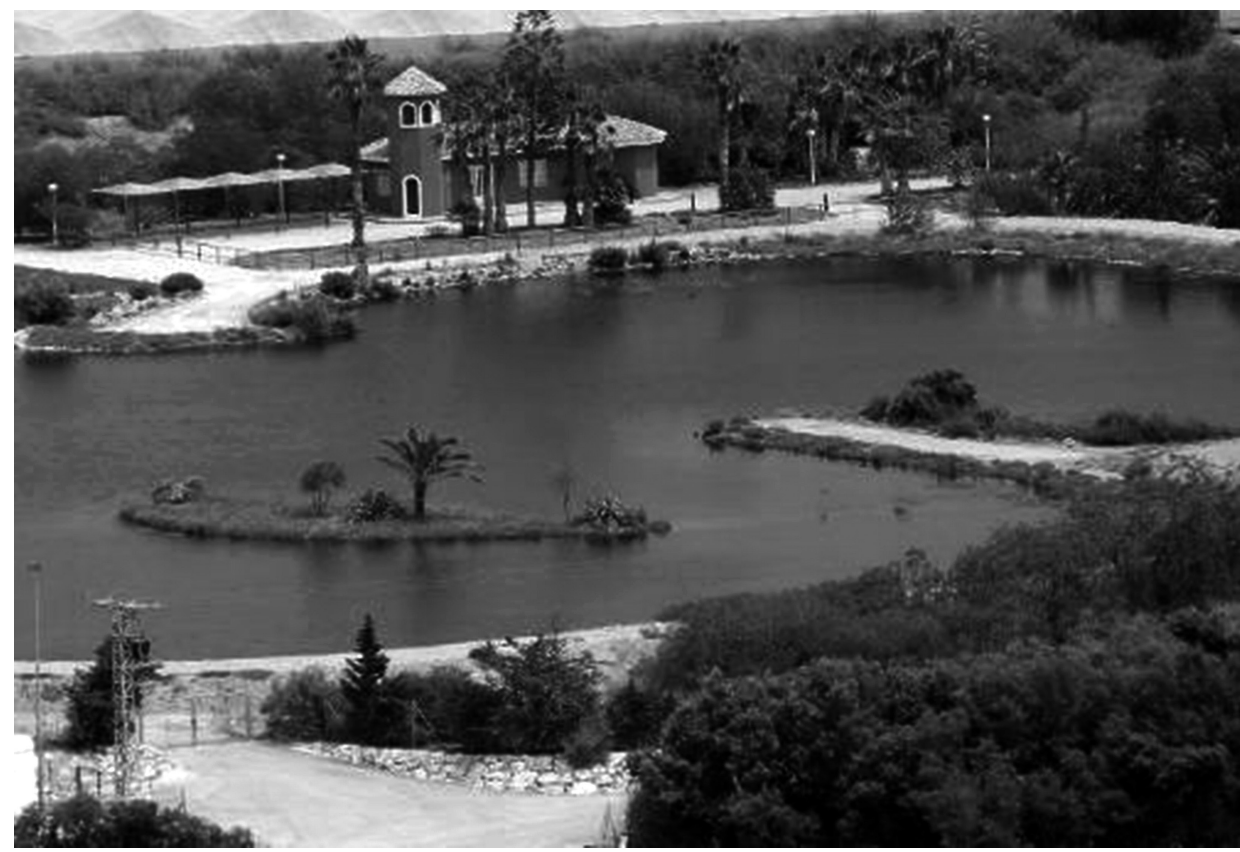

Fuente: archivo Dirección General de Medio Ambiente de la Región de Murcia.

\section{Lagunas de Campotejar}

Las actuaciones principales de mejora paisajística y conservación ambiental fueron:

a) retirada de residuos, como aireadores y cableado de las antiguas lagunas depuradoras que suponían un impacto paisajístico importante.

b) colocación de dos islas de madera para facilitar la nidificación del Charrán común (Sterna hirundo) y para que sirvan como lugar de reposo a las aves acuáticas.

Se fijó con unos anclajes pesados al fondo de la laguna, con cable de acero inoxidable y lastres de hormigón sulforresistente de $125-150 \mathrm{~kg}$ de peso. Las plataformas de madera están fabricadas por material flotante formado por 10 tubos cerrados de PVC de $\varnothing 20 \mathrm{~cm}$ estancos con una base de madera de 3,00 
x 3,00 m sobre un sistema de flotación. Finalmente se le dotó de una rampa y dos zonas de sombra a cada plataforma. Sobre la plataforma se puede disponer tierra, vegetación, etc.

La base es de madera tratada con sistema de autoclave que la protege del contacto directo con el agua, la humedad constante y la intensa insolación (Figura 9).

\section{FIGURA 9}

\section{PLATAFORMA FLOTANTE PARA NIDIFICACIÓN DE AVES}

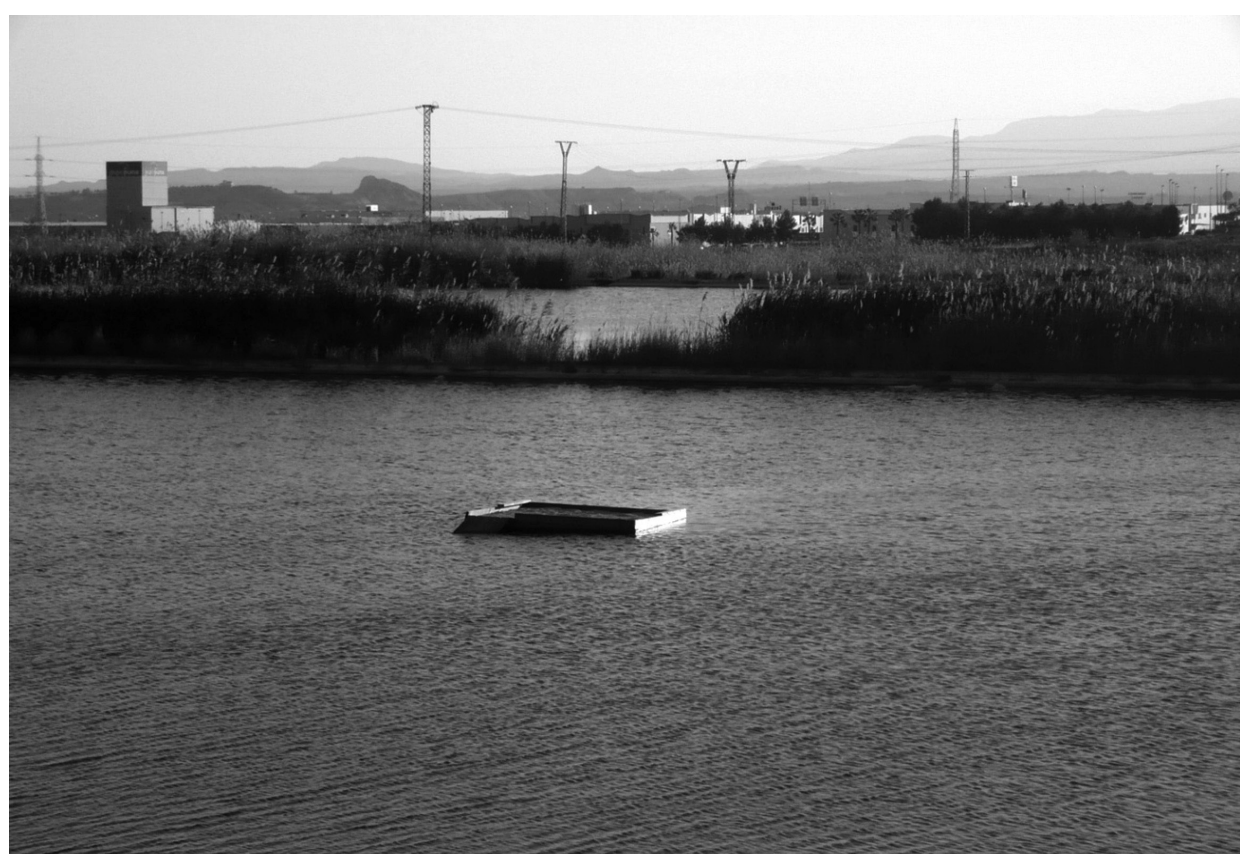

Fuente: elaboración propia. Situada en el centro del humedal, se instaló para que fuera utilizada como lugar de descanso y reproducción de determinadas especies de aves acuáticas.

c) dos reductores de velocidad a lo largo del vial que comunica la entrada a las lagunas con la EDAR para minimizar el número de vertebrados atropellados.

d) colocación de una pantalla de amortiguación acústica y paisajística dentro del recinto de las Lagunas, ya que cerca de la laguna 3 y junto al edificio de mantenimiento, hay una zona vallada que contiene motores de una 
de las comunidades de regantes que utilizan el agua de la depuradora, que representan un impacto acústico y paisajístico muy importante. La pantalla tiene una longitud total de 65 metros lineales y es de cañizo de caña natural, de $2 \mathrm{~m}$ de altura, tejido con alambre galvanizado, suministrado en rollos y sujeto con alambre galvanizado sobre el vallado existente.

e) recuperación de una laguna vacía mediante su conexión con otra laguna. El complejo lagunar de Campotejar en Molina de Segura se encuentra compuesto por 5 lagunas, de las que una se encontraba vacía, por lo que se instaló una tubería de distribución de agua con otra laguna (Figura 10).

\section{Figura 10}

TUBERÍA DE DISTRIBUCIÓN DE AGUA ENTRE LAS LAGUNAS

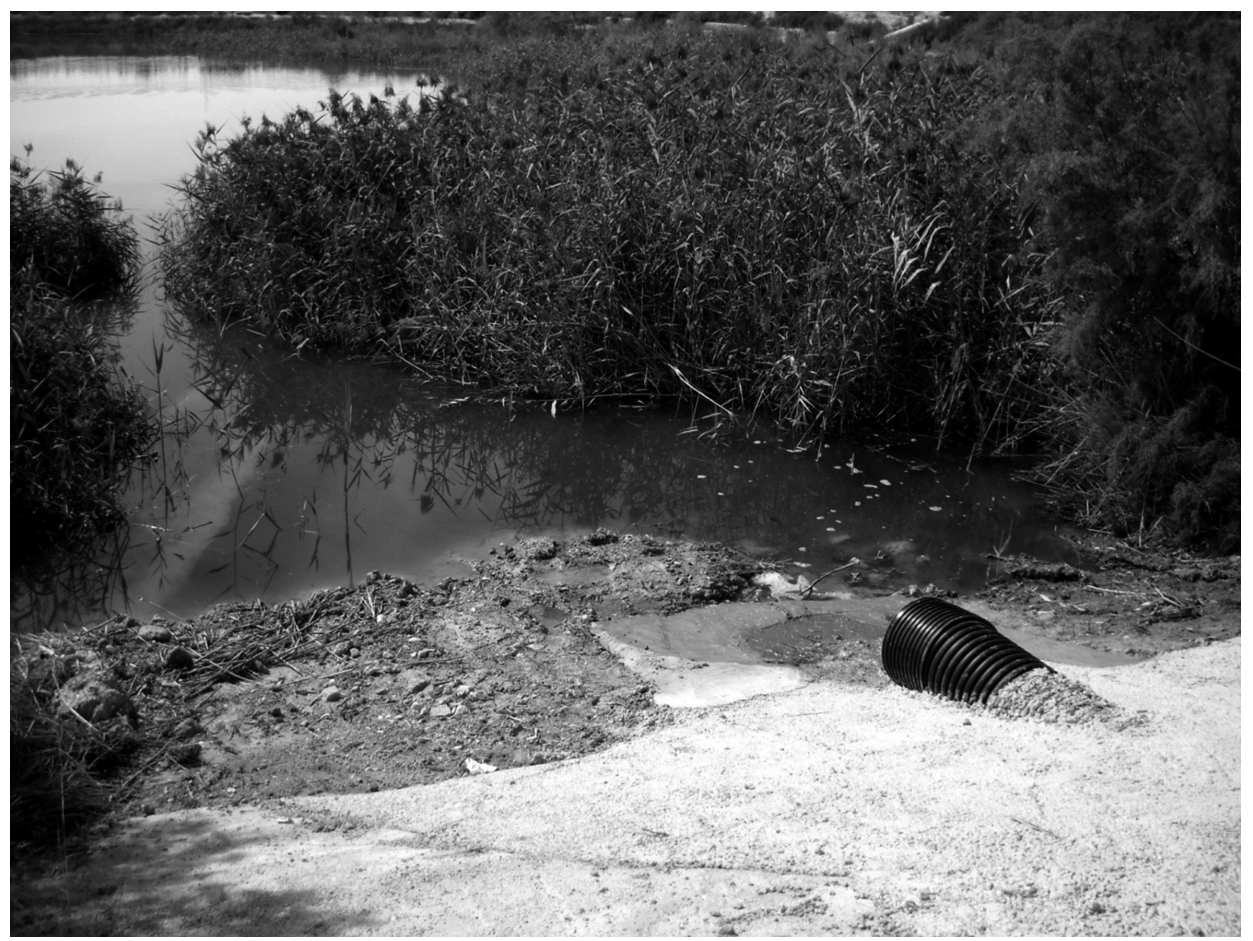

Fuente: elaboración propia.

Estudios Geográficos, Vol. LXXIX, 285, pp. 397-417, julio-diciembre 2018 ISSN: 0014-1496, eISSN: 1988-8546, doi: https//doi.org/10.3989/estgeogr.201815 


\section{DiSCUSIÓN Y CONCLUSIONES}

La depuración de aguas residuales mediante modernas depuradoras, sus usos posteriores, principalmente agrícola, y los valores naturales que bajo determinadas circunstancias pueden llegar a aglutinar, demuestra que estas lagunas artificiales pueden llegar a cumplir una doble función: una función social y económica al actuar como reservorios de agua para regadío y potenciar el desarrollo de actividades de turismo de la naturaleza y educación ambiental; y, por otro, una función ecológica, como humedales de agua dulce o salobre donde se desarrollen hábitats de gran interés ecológico por la presencia de especies de invertebrados y vertebrados que pueden llegar a estar amenazadas en el contexto regional, nacional e incluso internacional.

Los bienes y servicios proporcionados por éstos humedales pueden ser considerados indicadores de un modelo de sostenibilidad, que compatibiliza la regeneración de un recurso muy escaso en determinadas regiones desérticas como la Región de Murcia (el agua), con su aprovechamiento económico (agricultura irrigada) y social (turismo, educación ambiental), y con la diversificación de un ambiente natural semiárido (nuevos hábitat acuáticos) con reconocimiento internacional.

El hecho de que estos humedales, con estructura y alimentación artificial, puedan llegar a ser enclaves de importancia internacional, entre otros valores naturales, por determinadas especies de aves acuáticas, está directamente asociado a la sustitución del sistema de depuración por lagunaje, implantado en la geografía mediterránea ibérica a lo largo de los años 80, y hoy obsoleto, por sistemas de alta tecnología que permiten obtener agua adecuada para riego, su almacenamiento en balsas de la antigua estación depuradora de aguas residuales y su posterior naturalización.

El modelo de sostenibilidad, consistente en la depuración del aguaconservación de la naturaleza-explotación del recurso hídrico, que se ha generado por la mano del hombre en éstos humedales, puede ser considerado como modelo para impulsar iniciativas similares en instalaciones donde sistemas de depuración más modernos y eficaces sustituyen a obsoletos sistemas por lagunaje, contribuyendo así a la conservación del territorio por la ampliación de la red de humedales y el consiguiente enriquecimiento de la biodiversidad.

Una vez ejecutadas las actuaciones sobre la cubeta, garantizado el suministro hídrico y una adecuada cobertura de vegetación perimetral, se debería abordar la integración paisajística, incluyendo la eliminación de especies exóticas invasoras y la puesta en valor del edificio de la antigua depuradora de lagunaje de las Moreras como aula de educación ambiental (edificio de las Figuras 7 y 8 ). 
BiBLIOGRAFÍA

Ballesteros, G. A. y Muñoz, A. (2011a): Ficha de Información Ramsar de las Lagunas de Campotejar, Consejería de Agricultura y Agua de la Región de Murcia, 21 pp.

Ballesteros, G. A. y Muñoz, A. (2011b): Ficha de Información Ramsar de las Lagunas de las Moreras, Consejería de Agricultura de la Región de Murcia, 15 pp.

Ballesteros, G. A. (2015): Censos coordinados de Oxyura leucocephala (2004-2015), Consejería de Agricultura, Agua y Medio Ambiente de la Región de Murcia.

Crivelli, M. (1994): Characteristics of Mediterranean wetlands, Arles, Francia, MedWet/ Tour du Valat Publication, 1, 90 pp.

Comité de Humedales (2011): Protocolo de Inclusión de Humedales Españoles en la Lista de Importancia Internacional (Convenio de Ramsar) y Anexo Técnico, Madrid, Ministerio de Medio Ambiente, Medio Rural y Marino, 76 pp.

Esamur [Entidad de Saneamiento y Depuración de Aguas Residuales de la Región de Murcia] (2015): Aguas residuales en la Región de Murcia, Entidad de Saneamiento y Depuración de la Región de Murcia, http://www.esamur.com (Fecha de consulta: 26/4/2017).

Madroño, A., González, C. y Atienza, J. C. (eds.) (2005): Libro Rojo de las Aves de España, Madrid, DGB (MIMAM) y SEO/BirdLife, 452 pp.

Ministerio de Medio Ambiente (2007): El Plan Nacional de Calidad de las Aguas: Saneamiento y Depuración 2007-2015, Ministerio de Medio Ambiente de España, $117 \mathrm{pp}$.

Moreno, J. L., Millán, A., Suárez, M. L., Vidal-Abarca, M. R. y Velasco, J. (1997): "Aquatic Coleoptera and Heteroptera assemblages in waterbodies from emphemeral coastal streams («ramblas») of south-eastern Spain”, Archiv fuer Hidrobiologie, 141, pp. 93-97.

Sánchez-Fernández, D., Abellán, P., Velasco, J. y Millán, A. (2003): Los coleópteros acuáticos de la Región de Murcia. Catálogo faunístico y áreas prioritarias de conservación, Monografías de la S.E.A. Sociedad Entomológica Aragonesa, 10, Zaragoza, Sociedad Entomológica Aragonesa, SEA, 70 pp.

Wetlands International (2006): Waterbird population estimates, 4th edition, Wageningen, Wetlands International, $239 \mathrm{pp}$.

Fecha de recepción: 29 de septiembre de 2017.

Fecha de aceptación: 2 de octubre de 2018. 